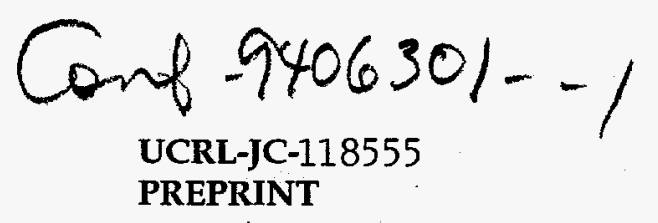

\title{
Issues Relevant to the Development of Coupled Chemistry/Climate Models
}

\author{
J.E. Penner ${ }^{1}$, J. Austin ${ }^{2}$, D. Cariolle ${ }^{3}$, H. Kelder ${ }^{4}$, A. Kylling ${ }^{5}$, M.J. Prather ${ }^{6}$, B. Steil ${ }^{7}$, \\ J.K. Sundet 8 \\ ${ }^{1}$ Lawrence Livermore National Laboratory, Livermore, CA 94550, USA. ${ }^{2}$ Meteoro- \\ logical Office, Bracknell, Berkshire, RG12 2SZ, UK. \\ ${ }^{3}$ Centre National de Recherches Meteorologiques, Toulouse, Cedex 31507, France. \\ ${ }^{4}$ Royal Netherlands Meteorological Institute, 3730 AE de Bilt, Wilhelminalaan 10, \\ Netherlands. \\ 5Institute of Mathematical and Physical Science, Dept. Physics, University of Tromsö, \\ N-9037, Tromsö Norway. \\ 6University of California at Irvine, School of Physical Sciences, Irvine, CA 92717, \\ USA. \\ ${ }^{7}$ Max-Planck-Institut für Chemie, Division of Atmospheric Chemistry, Postfach 3060 , \\ 55020 Mainz, Germany. \\ ${ }^{8}$ Institute of Geophysics, University of Oslo, PO Box 1022, Blindern, Oslo 0315, \\ Norway.
}

Discussion paper prepared at the NATO Advanced Research Workshop

"Atmospheric Ozone as a Climate Gas"

Lillehammer, Norway, 19-23 June 1994.

September 1994

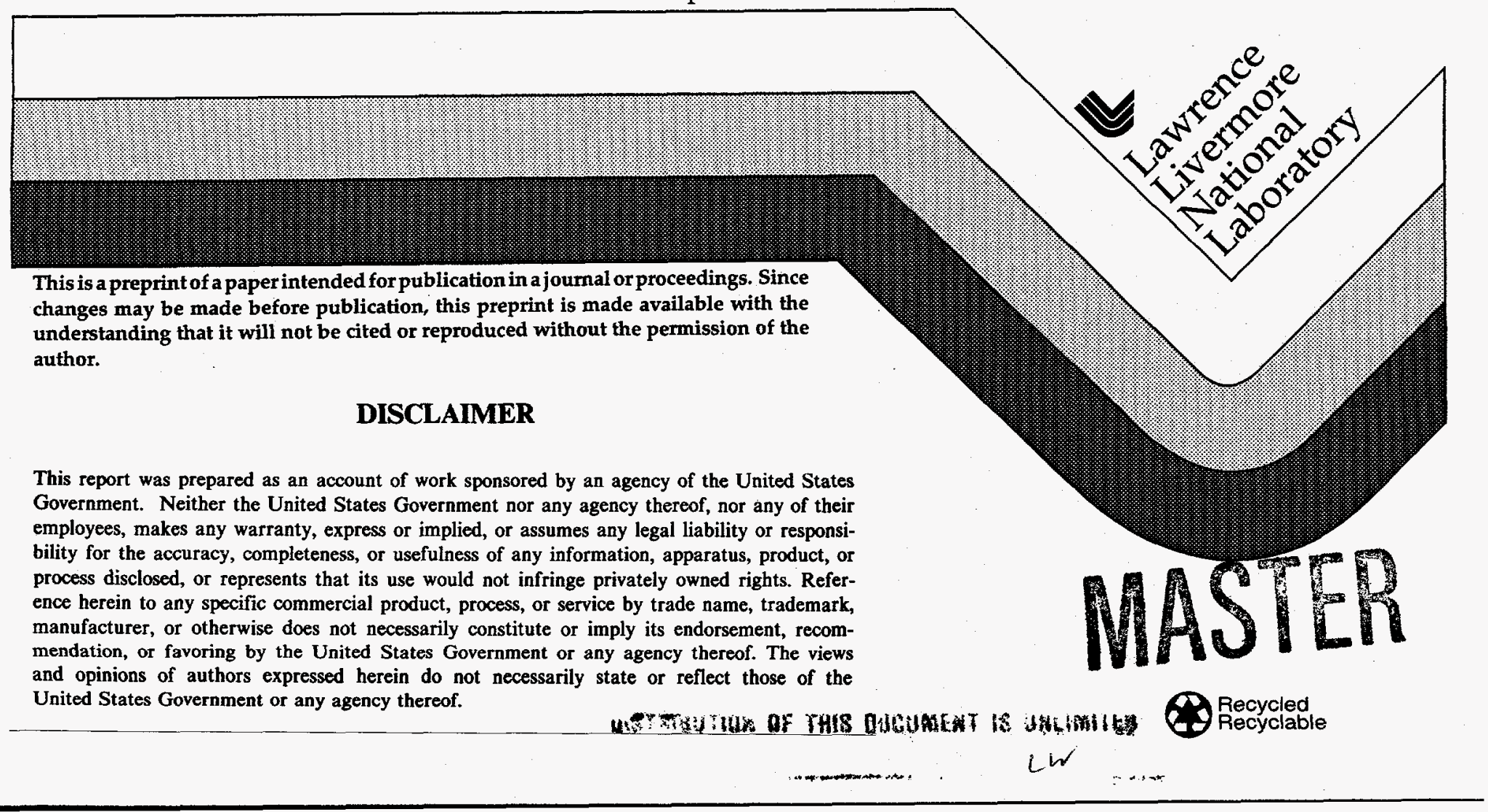




\section{DISCLAIMER}

This document was prepared as an account of work sponsored by an agency of the United State Government. Neither the United States Government nor the University of California nor any of their employees, makes any warranty; express or implied, or assumes any legal liability or responsibility for the accuracy, completeness, or usefulness of any information, apparatus, product or process disclosed, or represents that its use would not infringe privately owned rights. Reference herein to any specific commercial products, process, or service by trade name, trademark, manufacturer, or otherwise, does not necessarily constitute or imply its endorsement, recommendation, or favoring by the United States Government or the University of California. The views and opinions of authors expressed herein do not necessarily state or reflect those of the United States Government or the University of California, and shall not be used for advertising or product endorsement purposes. 


\section{DISCLAIMER}

Portions of this document may be illegible in electronic image products. Images are produced from the best available original document. 
J.E. Penner ${ }^{1}$, J. Austin ${ }^{2}$, D. Cariolle ${ }^{3}$, H. Kelder ${ }^{4}$, A. Kylling 5 , M.J. Prather ${ }^{6}$, B. Steil ${ }^{7}$, J.K. Sundet 8

1Lawrence Livermore National Laboratory, Livermore, CA 94550, USA. 2 Meteorological Office, Bracknell, Berkshire, RG12 2SZ, UK.

${ }^{3}$ Centre National de Recherches Meteorologiques, Toulouse, Cedex 31507, France.

${ }^{4}$ Royal Netherlands Meteorological Institute, 3730 AE de Bilt, Wilhelminalaan 10, Netherlands.

5Institute of Mathematical and Physical Science, Dept. Physics, University of Tromsö, N9037, Tromsö Norway.

6University of California at Irvine, School of Physical Sciences, Irvine, CA 92717, USA.

${ }^{7}$ Max-Planck-Institut für Chemie, Division of Atmospheric Chemistry, Postfach 3060,55020 Mainz, Germany.

8Institute of Geophysics, University of Oslo, PO Box 1022, Blindern, Oslo 0315, Norway.

Discussion paper prepared at the NATO Advanced Research Workshop

"Atmospheric Ozone as a Climate Gas"

Lillehammer, Norway, 19-23 June 1994.

\section{Introduction}

Our belief that climate will inexorably change is driven by the very clear evidence of chemical abundance changes in the atmosphere during the last four decades (Houghton et al., 1990, 1992). In the past, this belief has led to the development of two separate efforts: to understand and build models to describing the physical climate system and to understand and build models of the chemical climate system. But how will these two systems interact? Are there important chemical changes that result from climate change that need to be understood and properly accounted for in order to predict the evolution of the climate system? Are there important climate changes that may affect chemical abundances that must be addressed?

Examples of important chemical/climate interactions, involving ozone as well as other gases and aerosols, already exist in the published scientific literature. For example, it is known that ozone has been decreasing in the lower stratosphere since the 1980 s and increasing in the troposphere at northern mid-latitudes since the pre-industrial era (WMO, 1991). Calculations have shown that changes in the vertical distribution of ozone can lead to positive or negative radiative forcing, depending on the altitude of increase or decrease (Wang and Sze, 1980; Lacis et al., 1990). Calculations which adopt the measured changes in the vertical distribution of ozone over a recent decadal time period show that the radiative forcing from these changes 
is positive and, moreover, it is comparable to that due to increases in $\mathrm{CO}_{2}, \mathrm{CH}_{4}$, the chlorofluorocarbons (CFC-11, CFC-12), and $\mathrm{N}_{2} \mathrm{O}$ over the same period (Wang et al., 1993). Similarly, calculations have demonstrated that the negative climate forcing from photochemically generated sulfate aerosols is important relative to the positive forcing predicted for $\mathrm{CO}_{2}$ and other greenhouse gases (Kiehl and Briglieb, 1993; Taylor and Penner, 1994). These changes to radiative forcing by ozone and aerosols are not generally taken into account in current long-term simulations of climate change.

Changes in the climate system may also affect chemical abundances. For example, it has been understood for over a decade that decreases in stratospheric temperature forced by a $\mathrm{CO}_{2}$ doubling would reduce the rates of $\mathrm{O}_{3}$ chemical loss, thereby increasing stratospheric ozone. Increases in $\mathrm{CO}_{2}$ could also cause increases in polar stratospheric clouds (PSCs) during extremely cold winters, greatly enhancing the chlorine-catalyzed destruction of ozone with the possibility of forming an Arctic ozone hole (Austin and Butchart, 1994; Cariolle, this volume). As another example, increases in convective activity are expected to lead to increases in lightning frequency (Price and Rind, 1994). Since lightning provides an important source of $\mathrm{NO}_{\mathrm{x}}$ to the upper troposphere, such increases are expected to lead to increases in upper tropospheric $\mathrm{NO}_{\mathbf{x}}$, thereby increasing an important ozone precursor species. Further changes may be speculated, but are not yet quantified. Examples include the following: changes in cloudiness possibly altering the rates of heterogeneous processes that affect the global abundance of $\mathrm{O}_{3}$ and $\mathrm{NO}_{\mathrm{x}}$ (Lelieveldt and Crutzen, 1990); changes in precipitation leading to changes in the removal of pollutants that either contribute to tropospheric ozone formation (e.g., $\mathrm{NO}_{\mathrm{x}}$ ) or to ozone destruction in the absence of sufficient $\mathrm{NO}_{\mathrm{x}}$ (e.g., $\mathrm{HO}_{2}$ ); and changes to the biosphere resulting from climate change leading to important changes in chemically important emissions from the biosphere (e.g. isoprene, $\mathrm{NO}_{\mathbf{x}}$, $\mathrm{N}_{2} \mathrm{O}$, and $\mathrm{CH}_{4}$ ).

These examples point to the need to develop a coupled chemistry/climate model in order to successfully predict the evolution of the climate system. What issues need to be addressed in order to couple today's chemistry models with today's climate models? What uncertainties exist in the chemical models that are likely to affect our ability to predict future climates? What uncertainties exist in the climate models that may impact our ability to predict the future evolution of chemical abundances?

In section 2 , we outline which variables from the climate system are important to the chemical system and vice versa. We then explore in section 3 those problems in existing chemistry and climate models which may impact adequate quantification of the coupled chemistry/climate system. In section 4 , examples are provided where a consistency of 
treatment of variables in the two models is considered most important and why such variables should be treated in a like manner. In section 5 , a strategy is outlined for beginning to quantify the possible errors associated with these problems or with inadequacies in the coupling. Sections 6 and 7 give our estimates of the present level of accuracy in chemical transport models and general circulation models, respectively, and, in section 8 , some specific recommendations are given for near-term projects to build upon today's models in order to reach the goal of a predictive coupled chemistry/climate model. The conclusion is given in section 9 .

2. Variables Required by the Interacting Chemistry-Climate Modules

The major variables or processes which affect the interaction between individual chemistryclimate components are schematically illustrated in Figure 4.1. Starting from the General Circulation Model (GCM), the wind fields (u, v, w) are required to calculate the chemical transport terms. The specific humidity (q) is necessary to calculate the odd hydrogen species $\left(\mathrm{H}, \mathrm{OH}, \mathrm{HO}_{2}\right)$, which play a major role in tropospheric photochemistry. Prediction of cloud liquid water and cloud evolution including the amount of air passing through the cloud is needed for the computation of cloud chemistry. Chemical rate coefficients are determined from the GCM temperature (T) while the albedo and cloud optical properties are necessary to compute photolysis rates. Convection plays a significant role in rapidly transporting chemical species from the Planetary Boundary Layer (PBL) into the upper troposphere. The PBL is a quasi-mixed region where the major chemical constituents have their main sources. The diurnal evolution of the PBL as well as dry and wet convection can then transport these species to the free troposphere. In the stratosphere, where a separate photochemical module may operate, the horizontal wind fields are the main inputs from the GCM. Temperature is again important for the determination of the reaction rate coefficients and microphysical processes.

The Chemical Transport Model (CTM) output parameters which have a significant radiative effect are the greenhouse gases: $\mathrm{O}_{3}, \mathrm{~N}_{2} \mathrm{O}, \mathrm{CH}_{4}$, and CFCs. In some CTMs the heterogeneous chemistry component may also calculate the distribution of chemically produced aerosols (e.g., tropospheric sulfate aerosols) which provide a direct input to the radiation scheme and possibly to the cloud physics scheme in the GCM. For the stratosphere, the most important input to the GCM is the $\mathrm{O}_{3}$ amount. Stratospheric sulfate aerosols can play a significant role under volcanically perturbed conditions, and some models already include a separate aerosol module as part of the GCM itself. 
Many sources of greenhouse gases used in the CTM originate from the biosphere, the oceans and industrial activities and have feedbacks through the climate system. Processes in the biosphere are controlled by variables from the GCM (primarily temperature, precipitation, and photosynthetic active radiation) and from the CTM (primarily local ozone concentration and damaging solar UV). These biogeochemical processes are part of a "comprehensive" climate system, or an Earth System Model (ESM) but are not explicitly considered within the CTM. An important area which requires the addition of a biogeochemical model (BGM) is the prediction of future $\mathrm{CO}_{2}$ concentrations. The lifetime of $\mathrm{CO}_{2}$ in the atmosphere is not controlled by atmospheric chemical processes, but rather by biogeochemical processes in the terrestrial biosphere and by physical and biogeochemical processes in the ocean. As noted above, there are several other areas where emissions that involve the terrestrial or oceanic biosphere are important for either the climate system or the chemical system. For example, the emission of non-methane hydrocarbons (NMHCs) from trees increase during warm sunny weather. Also, excessive UV and high local ozone can damage vegetation, resulting in changes in biomass on decadal timescales. Increasing temperatures in the ocean may alter ocean productivity, thereby changing emissions of dimethylsulfide (DMS).

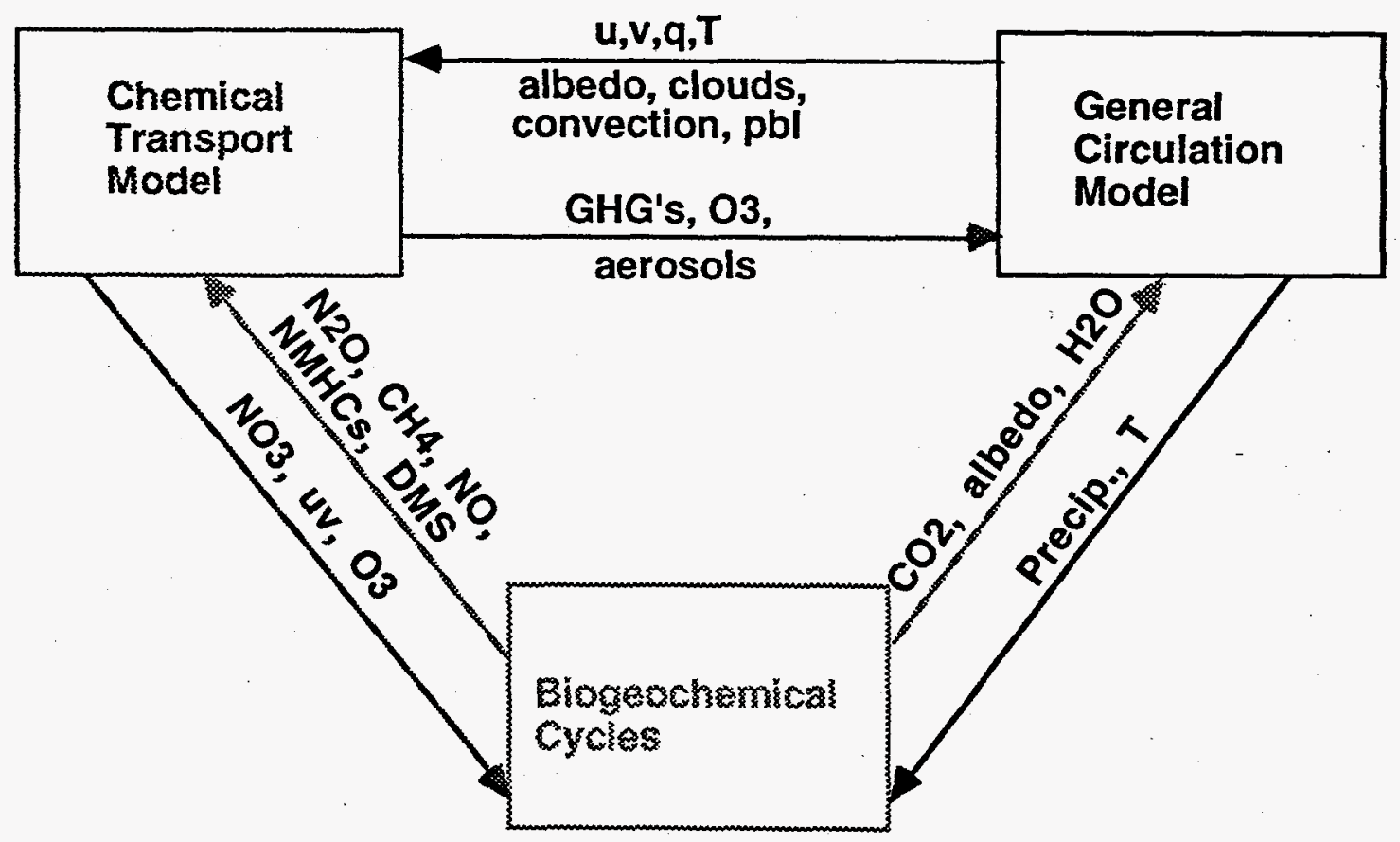

Figure 4.1 The chemistry/climate system may be thought of as having three components: a Chemical Transport Model, a General Circulation Model, and a Biogeochemical Cycles Model. The figure shows the prognostic variables from each of these models that are needed as input to the other models. 


\section{The current known problems with GCMs and CTMs}

(a) GCMs in the Stratosphere

During the past five years there has been substantial progress in the extension of GCMs into the stratosphere. These models now include the relevant physical processes important for the study of the upper atmosphere and they are starting to be used to study the climate impact of stratospheric ozone depletion. Multi-year simulations at various horizontal resolutions, ranging from coarse $\left(5^{\circ}\right.$ latitude $\times 5^{\circ}$ longitude) to fine $\left(1.5^{\circ} \times 1.5^{\circ}\right)$, have been performed allowing a better view of their strengths and weaknesses (Mahlman and Umscheid, 1987; Déqué et al., 1994). Most models can now reproduce the main characteristics of the extratropical stratospheric dynamics: a Northern Hemisphere (NH) winter perturbed by minor and major warmings, and a more zonal flow in the Southern Hemisphere (SH) winter with polar temperatures $20^{\circ} \mathrm{K}$ colder than in the NH.

The effect of the large $\mathrm{O}_{3}$ depletion following the build up of an Antarctic ozone hole has also been studied. The atmospheric thermal response with a large cooling inside the SH polar vortex in October and a warming at the stratopause level has been successfully reproduced. An increase in the duration of the SH polar vortex by about two weeks has also been successfully reproduced (Kiehl, et al., 1990; Cariolle et al., 1990).

There are still, however, a number of problems and deficiencies that are particularly important in the context of coupling these models with CTMs. One of them is the systematic tendency for the model to produce a polar vortex which is too cold, by a few degrees inside the $\mathrm{NH}$ vortex, but by more than $10^{\circ} \mathrm{K}$ in the SH. Given the extreme sensitivity of heterogeneous processes to temperature, forcing or coupling a CTM with a GCM having such a cold bias could lead to unrealistic present day climate simulations with a long lasting ozone hole in the SH and extensive ozone depletion through early spring in the NH (Austin and Butchart, this volume). This cold bias problem clearly impairs realistic coupled simulations. Preliminary results seem to indicate that the parameterization of the effect of gravity wave breaking on the momentum budget of the stratosphere needs to be refined. In addition to orographic waves, other sources of waves like those produced by convection could play an equally important role in decelerating the SH polar winter jet leading to adiabatic warming of the polar air masses. This result must be confirmed but there is a reasonable hope that the cold bias problem may be resolved within the next few years.

Another important problem concerns the apparent lack of interannual variability found in model simulations. For instance, extreme events like very early or very late warmings in the 
NH (Labitzke, 1986) or modulation of the SH polar temperatures by the quasi-biennial oscillation (QBO) are not well reproduced. In addition, regular wind regimes found at the equator are not fully developed, like the semi-annual oscillation in the upper stratosphere. The simulation of the QBO is either non-existent or has a very weak amplitude (Cariolle et al., 1993). It is not clear at present to what extent these deficiencies would impact the ability of a coupled GCM/CTM to predict climate evolution over several decades. Another difficult problem concerns the representation of the tropopause by the GCM. Present models do not resolve the sharp transition of dynamical regimes at the tropopause. This means that the CTM predictions of $\mathrm{O}_{3}$ change at that level would not be adequately taken into account by the GCM. In addition to these problems, temperature deficiencies near the tropopause directly impact the calculated water vapor distribution at that level and in the stratosphere above. At high latitude near the $200 \mathrm{hPa}$ level the GCMs produce a very dry atmosphere due to their tendency to underestimate the temperature by $5^{\circ} \mathrm{K}$ or more. At the equatorial tropopause the ability to model a mean temperature close to the observed might not even be sufficient since the drying process appears to require the occurrence of extreme small-scale cold events associated with deep convection. For these reasons and also because of the limited vertical resolution possible with GCMs, the modeled water vapor distribution is uncertain and should be used with caution within the CTM. If we want to take better account of dynamical and radiative processes at the frontier between troposphere and stratosphere, it might be necessary to increase substantially the vertical resolution of the GCM at the tropopause level.

Despite all these problems coupled GCM/CTMs have been integrated either with simplified parameterizations or with reasonably comprehensive chemical codes. With simplified parameterizations it has been possible to reproduce the seasonal march of the stratospheric $\mathrm{O}_{3}$ distribution. Coupled models with more comprehensive chemical codes have already revealed difficulties in coupling the ozone chemistry with the climate model, but have not yet been integrated for long enough periods to assess all possible deficiencies.

(b) GCMs in the Troposphere

The main features of the tropospheric circulation are well reproduced by GCMs, but significant differences between model and observations can exist at the regional scale. For example, the monsoon circulation is sometimes shifted in latitude and develops with a different timing compared to observations. The blocking sequences over the Atlantic and Pacific regions are often underpredicted by the GCM so that meridional transport is less intense than observed in those regions. 
The largest uncertainties in the troposphere are related to the parameterization of sub-grid phenomena, notably clouds and convection. Often, these are the processes used to "tune" GCMs so that larger scale predicted features match observations. It is difficult to identify specific errors without substantial verification of sub-grid processes through the use of high resolution models combined with observations. In particular, low clouds over the ocean in anticyclonic conditions and in regions with large vertical stability are not well reproduced. This could impact chemistry via lack of cloud processing and an excess of radiation.

(c) CTMs in the Stratosphere

CTM simulations of the stratosphere have some known systematic errors associated with photochemistry, such as the underprediction of ozone in the upper stratosphere, but clear examples of errors in the combined chemistry and transport are more difficult to document. There is reasonable consensus that a quantitative explanation of the overall depletion of total ozone over the Antarctic is now possible (Solomon, 1990). Nonetheless, fully coupled model simulations are sensitive to the $\mathrm{HNO}_{3}$ saturated vapor pressures assumed in the vicinity of PSCs and in some cases may calculate ozone holes for relatively low levels of total chlorine. In mid-latitudes total ozone has decreased significantly during the last decade (Stolarski et al., 1991). This has been attributed to heterogeneous chemistry on background aerosol but has not yet received a quantitative explanation.

The reaction rate coefficients for gases are reasonably well known (DeMore et al., 1992), particularly those which have been measured over the full range of stratospheric temperatures. There are, however, greater uncertainties in 3-body reaction rate coefficients, particularly for the lower stratospheric temperature regime where the rates are generally faster than at higher temperatures and the laboratory measurements are fewer. The representation of heterogeneous (i.e. gas-aerosol) processes has improved significantly in recent years (DeMore et al., 1992). Based on laboratory studies, models and observations, it is known that key heterogeneous processing appears at certain threshold temperatures. Thus temperature errors of several degrees are only important near this threshold. Further discussion of uncertainties in stratospheric chemistry is given in Chapter 2.

(d) CTMs in the Troposphere

Chapter 2 has described many of the uncertainties in the chemical processes and their consequences for modeling tropospheric ozone: for example, the partitioning of $\mathrm{NO}_{\mathrm{y}}$, the odd-hydrogen budget, the role of aqueous and surface reactions, and the oxidation products of NMHCs. Here, we specifically address issues related to the use of GCM variables to 
predict rates of chemical processing in the troposphere. The primary issue of concern in this respect is the lack of resolution provided by most GCMs. For example, it is well known that the net production of ozone depends critically on $\mathrm{NO}_{\mathrm{x}}$ concentrations. If the $\mathrm{GCM}$ grid cannot resolve the emissions of plumes associated with different sources, it may be necessary to develop parameterizations to treat the chemistry of these plumes on sub-grid scales. Similarly, if the GCM can only predict the fraction of a grid box covered by clouds, it will be necessary to develop a parameterization to account for how much air flows through the subgrid cloud area in order to predict rates of chemical reactions within clouds. Pollutant transport through convection is another area where parameterization is needed. The GCM transport by convection is generally developed to give physically realistic transport of heat and momentum. But the CTM needs to parameterize the transport of insoluble species, soluble species, and aerosols, each of which may have a different transport efficiency, depending on the amount and type of precipitation developed during the convective event. Systematic errors in these "chemical" treatments will lead to changes in the prediction of future greenhouse gases, such as $\mathrm{O}_{3}$. We recommend that the uncertainty of treatment by these parameterizations be thoroughly explored so that a range of results from the CTM might be provided to the GCM.

\section{Regions Where Consistency is Important}

A consistent treatment of variables passed between the CTM and GCM is very important in regions where strong gradients occur both in dynamical and chemical properties. The main regions of concern are the planetary boundary layer, the tropopause, and the polar vortices. As coupled CTMs and GCMs are developed it will be important to quantify whether lack of resolution in these regions may result in important inaccuracies that affect the coupled system.

(a) The boundary layer

From a dynamical point of view there is a separation between a region of rapid vertical mixing in the PBL and that of slower mixing of the free troposphere. This can significantly affect chemical modeling because boundary layer emissions can be rapidly converted and could form different product yields if they were confined together in small volumes. Significant exchange between the boundary layer and the free troposphere occurs during convection and in extratropical cyclones (Browell et al., 1987; Allaart et al., 1992). A clear mismatch between GCM and CTM may occur when the CTM predicts aerosol formation in the boundary layer and feeds this information back into the GCM calculation of radiation and 
clouds. In this case it is critical that the aerosols are not misplaced within the GCM's boundary layer or the radiative forcing and effects on clouds may be misplaced.

(b) The tropopause

The tropopause separates the troposphere, where vertical mixing takes place in days, from the stratosphere, where vertical mixing takes place over periods of weeks to months. Its position may be determined by gradients of temperature or potential vorticity. In tropical regions convective events with time scale of hours and horizontal scales of 10 to $1000 \mathrm{~km}$ appear to inject tropospheric air into the stratosphere. The return exchange generally takes place in tropopause-folding events driven by cyclones and convection in the extra tropics (Danielsen and Mohnen, 1977; Allaard et al., 1994). The very sharp gradient of $\mathrm{O}_{3}$ across the tropopause results in corresponding large gradients in the solar heating rates. Furthermore CTM predictions of the sign of $\mathrm{O}_{3}$ perturbations in this region depend critically on their location relative to the tropopause. An unanswered question is at what scales the chemical gradients must be preserved during the mixing process in order to correctly predict the $\mathrm{O}_{3}$ perturbations.

\section{(c) Polar vortices}

The edge of the circumpolar vortex may be determined by the region of strongest winds surrounding the pole. To a first approximation this region isolates air inside the vortex from air outside. Temperatures inside the vortex may be low enough to produce PSCs which lead to $\mathrm{O}_{3}$ depletion. Steep ozone gradients may then be established across the vortex edge and result in correspondingly sharp gradients in local solar heating rates. Exchange across this boundary is slow in mid winter and occurs through reversible and irreversible deformation by planetary waves, filamentary peeling, and small scale exchange caused by waves and turbulence (Plumb et al., 1993). Dynamical maintenance of the vortex is enhanced by polar ozone loss as discussed above, so that the chemical depletion predicted by the CTM must be accurately represented relative to the vortex edge in the GCM.

\section{A Strategy for Assessing Propagation of Errors in a Coupled CTM/GCM}

Both GCMs and CTMs are highly non-linear, and furthermore, the meteorology in the GCM is inherently chaotic. The coupled model, consisting of both a GCM and a CTM, is thus also non-linear, and errors from the GCM/CTM may amplify and lead to unrealistic states of the coupled system. Unfortunately, the onset of chaotic behavior in these systems obscures the 
physical processes responsible for the model errors. It is therefore desirable to devise methods to isolate and analyze processes in the absence of such chaos, and hence to identify errors that are of largest importance to the final predicted climate. Given the complexity, it is not feasible to study in a "brute force way" the effect of propagation of many individual errors within the fully coupled system. Hence, we must define a set of sensitivity tests that highlight potential problems with the coupling between the different parts of the full model. Future intercomparison studies between different coupled models may increase our understanding of the propagation of errors.

One strategy for beginning to quantify error propagation is to assign uncertainties to parameters exchanged between the different parts of the coupled system. Because a combination of errors may be hidden by canceling effects, the potential sensitivity to individual variables from GCMs and CTMs should be studied. It is important to realize that some variables may exhibit large errors but still have little effect on the coupled system and vice versa. Below we categorize levels of certainty in the variables predicted by the GCMs and CTMs to facilitate such a study.

\section{Levels of Certainty in CTM Predictions}

It is extremely difficult, and not necessarily meaningful, to place formal (i.e. statistical) uncertainties on each of the CTM outputs at this stage in their development. Rather, we choose to group the CTM outputs and assign a level of quality to each one, selecting three levels of quality:

Level 1: $\quad \pm 20 \%$ uncertainty--well known chemical cycles or processes;

Level 2: $\quad$ factor of 2 range--highly derived, uncertain processes;

Level 3: $\quad$ sign or magnitude change possible--many coupled, canceling terms, budget missing key terms.

The assignment of a particular CTM output to one of these categories is in large part subjective. It is based on an overall assessment of the adequacy of the CTM simulations. It includes both the accuracy of the physics and chemistry that go into the model (albeit in a highly simplified or parameterized form) and the critical evaluation of the CTM simulations compared with observations. For example, one critical CTM output is the concentration of greenhouse gases: $\mathrm{N}_{2} \mathrm{O}, \mathrm{CH}_{4}, \mathrm{CFCs}$, and others. The GCMs need the CTM output describing the distribution of these gases in order to calculate radiative forcing. The CTM calculation for $\mathrm{N}_{2} \mathrm{O}$ will predict a well mixed tropospheric abundance with fall-off in the stratosphere due to 
photochemical destruction. The tropospheric abundance is fairly uniform and depends on the mean atmospheric residence time of the gas (about 120 years), which involves integrating chemical losses over the globe. In contrast, the abundance in the upper stratosphere is a balance between transport and photochemical destruction on a time scale of a week. Thus, given the sources and atmospheric circulation, the CTM's ability to predict the free tropospheric abundance using the known chemical mechanism for the losses is very good (Level 1), but the predicted $\mathrm{N}_{2} \mathrm{O}$ abundances in the upper stratosphere, or in the Antarctic winter vortex, are no better than Level 2. Since the latter uncertainties are unimportant to the radiative forcing, the $\mathrm{N}_{2} \mathrm{O}$ values reported back to the GCM (i.e., only bulk tropospheric value with some measure of the stratospheric fall-off) are Level 1. Similar considerations apply to uncertainties in CFCs and the total $\mathrm{CH}_{4}$ abundance (but not necessarily to predicting small perturbations in $\mathrm{CH}_{4}$ ).

Some more uncertain predictions rely on less well understood processes, or involve several, often canceling effects. A key example here is the prediction of changes in $\mathrm{CH}_{4}$ or $\mathrm{O}_{3}$ abundance due to a complex scenario involving changes in $\mathrm{NO}_{\mathrm{x}}, \mathrm{CO}$, and $\mathrm{NMHCs}$. For example, the CTM prediction of changes since the pre-industrial era in $\mathrm{O}_{3}$, or even global mean $\mathrm{OH}$, are probably certain to a factor of 2 . Similar ranges apply to the CTM calculation of sulfate aerosols or the deposition of $\mathrm{NO}_{3}-$ and $\mathrm{SO}_{4}=$. Changes in stratospheric ozone due to climate changes and changes in CFC amounts are also considered to be categorized as Level 2. Currently, these uncertainties are probably just above $20 \%$ and arise from continuing uncertainties in the quantitative details of heterogeneous processes. For the next few decades, chlorine loading levels should decrease, which would reduce the influence of heterogeneous effects, but this might be canceled out by an increase in the radiative effect of $\mathrm{CO}_{2}$. Consequently, the sensitivity of polar ozone in the lower stratosphere to other atmospheric changes will probably be significant for the next 50 years or more.

Accurate predictions of changes in tropospheric ozone associated with small changes in emissions (e.g., the effect of aircraft fleet growth over the past decade imposed on other changes in global use of fossil fuel) are highly uncertain due to the possible non-linearities and the existence of canceling terms. Such predictions are considered to be only of Level 3 certainty. Also, for example, the formation of scattering aerosols and net production of $\mathrm{O}_{3}$ from the oxidation products of complex volatile organic compounds are highly uncertain because the source budgets are uncertain as well as the processes of chemical breakdown and aerosol formation.

We group the CTM outputs as follows: 
Level 1: $\quad \mathrm{N}_{2} \mathrm{O}, \mathrm{CFC}, \mathrm{CH}_{4}$ abundances

mean tropospheric and stratospheric $\mathrm{O}_{3}$

UVB changes

Level 2: $\quad$ changes in tropospheric $\mathrm{O}_{3}$ over the last 100 years

changes in stratospheric $\mathrm{O}_{3}$

sulfate aerosol concentrations

nitrate and sulfate deposition

Level 3: changes in tropospheric $\mathrm{O}_{3}$ from small, combined changes in $\mathrm{NO}_{\mathbf{x}}$,

NMHCs and $\mathrm{CH}_{4}$

organic aerosols

\section{Levels of Certainty in GCM Predictions}

The current generation of GCMs reproduces the main features of the atmospheric circulation (i.e. the zonal and meridional flow). The zonal component of the flow is well captured and despite a tendency for the flow to become more zonal when horizontal resolution increases the meridional circulation compares well with analyses. The temperature distribution is in reasonable agreement with observations except perhaps at the equatorial tropopause and at polar latitudes in the upper troposphere where the modeled temperatures are sometimes too low. Hence we place these variables in the Level 1 category. This does not imply that improvement in temperatures, for example, are not necessary, since the simulation of excessive ozone depletion is serious in the lower stratosphere (section 3a).

The distribution of the integrated water vapor content of the atmosphere given by satellite measurements agrees in general terms with model predictions, but regional differences can reach a factor 2 and quality of simulated $\mathrm{H}_{2} \mathrm{O}$ in the dry upper troposphere is not well known. This will impact the quality of CTM simulations due to the importance of water vapor as a precursor of $\mathrm{OH}$ radicals. Hence this variable is placed in the Level 2 category.

The fields giving the highest uncertainties describe subgrid scale processes with short lifetimes, and these couple directly with the calculated impact of short-lived species in the CTMs (e.g., the $\mathrm{NO}_{\mathrm{x}}$ and volatile organic compound distributions). Even less robust is the representation of clouds by the GCMs. Tunable parameters exist in the GCM predictions of clouds, the objective being to obtain a satisfactory radiative forcing by clouds. This does not cover the needs of the CTM, however, which requires knowledge of microphysical quantities like droplet and ice crystal size and distribution. It is inherently difficult to model clouds and convection given the nature of small scale circulation, even when they are obviously linked 
to the large-scale features like convergence and specific humidity that are resolved by the GCM. Convective processes are not described in a satisfactory way, with respect to either the spatial or temporal scales. The transport and deposition of momentum, mass and concentration remain highly uncertain. The global impact of processing of air in clouds is an area only newly considered. The impact on tropospheric ozone through reactions in droplets, including $\mathrm{HO}_{\mathrm{X}}, \mathrm{NO}_{\mathrm{x}}$, and even halogenated species, may be needed to give a more accurate ozone distribution (Jonson and Isaksen, 1993).

The boundary layer couples the large-scale circulation with the oceans and land surface. The representation of the non-slip lower boundary layer, giving the typical chaotic and coherent structure, makes both the evolution of the boundary layer and the net effect on the free troposphere regarding input of heat, moisture, momentum and trace species concentrations a source of large uncertainty.

In summary, we group the GCM outputs as follows:

Level 1: $\quad$ Resolved scale flow

Large scale temperature distribution

Level 2: Net mass flux to/from stratosphere-troposphere

The integrated water vapor

Level 3: $\quad$ Large Scale Clouds

Cumulus Clouds

Cloud processing

Boundary layer processes

\section{Near-Term Projects}

Here, we describe four near-term projects that could help to determine the importance of coupling in the chemistry/climate system and could help to delineate possible problems in the execution of this task. In the first two, we seek a broad sensitivity analysis to learn how important uncertainties in GCMs are for the predictions of CTMs. In the third, we seek to understand whether changes to $\mathrm{O}_{3}$ from preindustrial times to the present might be important to climate. In the fourth we define a strategy for testing resolution effects.

(a) Tropospheric Climate Project 
The Atmospheric Model Intercomparison Project (AMIP) has provided an excellent opportunity to compare GCMs. This project, coordinated by Gates (Program for Climate Model Diagnosis and Intercomparison, Lawrence Livermore National Laboratory, Livermore, CA, USA), has over 30 participating GCM modeling groups from around the world. Each group has used prescribed sea surface temperatures and sea ice as boundary conditions for a GCM simulation covering the period 1978-1989. The model results are being compared with each other and with analyzed model fields from numerical weather prediction models for the same time period, providing a sense of how well the climate variables can be predicted. The project provides an excellent opportunity to diagnose the response of CTMs that might result from uncertainties in GCMs. However, the output parameters that have been archived from these simulations are not sufficient to diagnose fully the problems and differences in chemical transport models that result from uncertainties in GCMs. Therefore, we recommend that an additional, possibly limited, sequence of experiments be performed, wherein several of the AMIP GCMs simulate the same time period, saving history files of the variables needed by the CTMs. The required variables are four dimensional fields of the variables pictured in Figure 4.1. These could then be used with a single or perhaps multiple CTMs to diagnose how important the differences in GCMs are for prediction of chemical abundances.

\section{(b) Stratospheric Climate Project}

For the stratosphere, an equivalent project to AMIP is currently being coordinated by Rood (NASA Goddard Space Flight Center, Greenbelt, MD, USA). While the proposal does not currently include a comparison of chemical consequences of differences in stratospheric circulation, we recommend that the proposed list of history file variables be scrutinized by stratospheric chemical modelers with respect to the variables identified here. A project could then be established to integrate stratospheric CTMs for sample periods within the stratospheric model intercomparison to investigate the sensitivity of stratospheric ozone predictions to differences in the stratospheric circulations of a range of GCMs.

\section{(c) Chemical Change Project.}

A sensitivity study for the GCMs based on CTM uncertainties in predicting changes in greenhouse gases, particularly ozone, should be defined. However, because of inherent differences between the GCM predictions of future climate (i.e., deterministic but chaotic) and the CTM predictions of changing atmospheric composition (i.e., repeatable), we must select a sensitivity study for the GCMs carefully. For modest perturbations in the radiative forcing of less than $1 \mathrm{~W} \mathrm{~m}^{-2}$, the GCMs would need to gather simulated climate statistics for more than several decades in order to detect differences with respect to a control run. Because such computer simulations are costly, we suggest that the CTM research groups 
pool their predictions of $\mathrm{O}_{3}$ change from pre-industrial to present and define a single "standard" prediction of change in $\mathrm{O}_{3}$ for GCMs to assess. The perturbations to $\mathrm{O}_{3}$ should emphasize the upper troposphere and lower stratosphere. It is important that this perturbation have a realistic magnitude, because we cannot confidently scale the predicted climate change for artificially large perturbations to that for a more realistic $\mathrm{O}_{3}$ change. (Previous studies of the greenhouse effect of $\mathrm{O}_{3}$ have often relied on exaggerating the ozone change in order to detect a statistically significant difference in climate after only a couple of decades.) This perturbation should be defined in absolute units of $\mathrm{O}_{3}$ change ( $\mathrm{ppb}$ rather than \%) and should include to first order the latitudinal and seasonal variations of ozone changes that are predicted to occur in the 3-dimensional CTMs.

A challenging part of this project is to define the $\mathrm{O}_{3}$ perturbation consistently with the tropopause structure of the GCMs (e.g. potential temperature and potential vorticity rather than in terms of geometric altitude). The typical chemical perturbation might have $\mathrm{O}_{3}$ increasing below the tropopause due to increasing fluxes of $\mathrm{NO}_{\mathbf{X}}, \mathrm{CH}_{4}, \mathrm{CO}$, and $\mathrm{NMHCs}$ and then decreasing above the tropopause due to enhanced $\mathrm{O}_{3}$ destruction from the breakdown of industrial halocarbons. It is critical for the climate impact of these changes that the sharp tropopause boundary in the CTM predictions be translated to radiative effects with similar change across the dynamical tropopause in the GCM. Establishing a method of achieving this consistency would be a major objective of this project.

(d) Mesoscale project

Two approaches to improving CTM modeling of subgrid scale chemical processing and transport are (1) use mesoscale models directly in a link between the CTM and GCM or (2) use high resolution studies with the mesoscale model to develop more accurate parameterizations within the GCM. Three features should be investigated by mesoscale models: (1) stratosphere/troposphere exchange processes, to determine the $\mathrm{O}_{3}$-abundance in the upper troposphere and lower stratosphere; (2) lee wave induced particle formation in the stratosphere, which is potentially of great influence in Arctic $\mathrm{O}_{3}$ destruction; and (3) convective processes which may be studied in mesoscale and cloud scale models to investigate mass transport and removal of trace species.

\section{Conclusions}

Large strides have been made in our capability to predict both the physical climate system and the chemical climate system during the past few years. In spite of extant uncertainties in both GCMs and CTMs, we should begin the process of understanding how and to what 
extent these errors might propagate in the coupled system. We have outlined a strategy for quantifying the effective uncertainties and have defined a few initial steps in the process. Systematic study of the importance of uncertainties and their impact on the coupled system is, we think, a prerequisite to progress in coupling the chemical and climate systems. Such coupling is necessary if we are to predict the evolution of climate in the future.

Acknowledgments. J.E.P. was supported by the US DOE Atmospheric Chemistry Program. Work at the Lawrence Livermore National laboratory is performed under the auspices of the U.S. Department of Energy under Contract W-7405-Eng-48. J.A. was partially supported by the UK DOE stratospheric change contract PEDC7/12/146. A.K. acknowledges support from the National Aeronautics and Space Administration through grant NAGW-2165 to the University of Alaska. M.J.P. acknowledges support from the NASA Atmospheric Chemistry program. B.S. was partially funded by DFG (German Science Foundation) and BMFT (Federal Ministry for Research and Technology). J.K.S was funded by the Norwegian Research Council, Nordic Ministry of Research.

\section{References}

Allaart M, Kelder H and Heijboer L (1992) Transport of trace gases by extratropical cyclones. Proc. Quadr. Ozone Symposium. Charlottesville. (in press)

Austin $J$ and Butchart N (1994) The influence of climate change and the timing of stratospheric warmings on Arctic ozone depletion. J Geophys Res 99: 1127-1145

Austin J and Butchart N (1995) Simulations of stratospheric ozone in a climate model. (this volume)

Browell E, Danielson E, Ismail S, Gregory G and Beck R (1987) Tropopause fold structure determined from air borne Lidar and in situ measurements. J Geophys Res 92: $2112-2120$

Cariolle D, Deque M, Lefevre F and Simon P (1994) GCM modeling of the stratospheric dynamics and its coupling with chemistry. (this volume)

Cariolle D, Amodei M, Deque M, Mahfouf JF, Simon P and Teyssedre H (1993) A quasibiennial oscillation signal in general circulation model simulations. Science 261 : $1313-1316$

Cariolle D, Lasserre-Bigorry A, Royer J-F and Geleyn J-F (1990) A GCM simulation of the springtime Antarctic ozone decrease and its impact on midlatitudes. J Geophys Res 95: 1883-1898

Danielsen E and Mohnen V (1977) Project dustorm report: Ozone transport, in situ measurements, and meteorological analysis of tropopause folding. J Geophys Res 87: $5867-5877$

Demore WB, Sander SP, Howard CJ, Ravishankara AR, Golden DM, Kolb CE, Hampson RF, Kurylo MJ and Molina MJ (1992) Chemical kinetics and photochemical data for use in stratospheric modeling. Evaluation number $10 \mathrm{JPL}$ 92-20

Déqué M, Devreton C, Braun A and Cariolle D (1994) The Arpege atmosphere model: A contribution to the French community climate modelling. Climate Dynamics. (in press)

Houghton JT, Jenkins GJ and Ephraums JJ (eds) (1990) Climate change in the IPCC Scientific Assessment. Cambridge University Press New York

Houghton JT, Callender BA and Valney SK (eds) (1992) Climate change 1992. In the Supplementary Report to the IPCC Scientific Assessment. Cambridge University Press New York

Jonson JE and Isaksen ISA (1993) Tropospheric ozone chemistry: The impact of cloud chemistry. J Atmos Chem 16: 99-122 
Kiehl JT, Boville BA and Briglieb BP (1988) Response of a general circulation model to a prescribed Antarctic ozone hole. Nature 332: 590-596

Kiehl JT and Briglieb BP (1993) The relative role of sulfate aerosols and greenhouse gases in climate forcing. Science 260: 311-314

Labitzke K (1986) On the interannual variability of the middle stratosphere during winter. In Handbook for MAP. Vol 18 S Kato (ed) pp 1-9 SCOSTEP

Lacis AA, Wuebbles DJ and Logan JA (1990) Radiative forcing of climate by changes in the vertical distribution of ozone. J Geophys Res 95: 9971-9982

Lelieveldt J and Crutzen PJ (1990) Influences of cloud photochemical processes on tropospheric ozone. Nature 343: 227-233

Mahlman JD and Umscheid LJ (1987) Comprehensive modeling of the middle atmosphere: The influence of horizontal resolution. In Transport Processes in the Middle. Atmosphere G Visconti and R Garcia (eds) D Reidel Norwell Mass.

Price C and Rind D (1994) Possible implications of global climate change on global lightning distributions and frequencies. J Geophys Res 99: 10823-10831

Solomon S (1990) Progress towards a quantitative understanding of Antarctic ozone depletion. Nature $347: 347-354$

Stolarski RS, Bloomfield P and McPeters RD (1991) Total ozone trends deduced from NIMBUS 7 TOMS data. Geophys Res Lett 18: 1015-1018

Taylor KE and Penner JE (1994) Response of the climate system to atmospheric aerosols and greenhouse gases. Nature 369: 734-737

Wang WC, Zhuang.YC and Bojkov RD (1993) Climate implications of observed changes in ozone vertical distributions at middle and high latitudes of the northern hemisphere. Geophys Res Lett 20: 1567-1570

Wang WC and Sze ND (1980) Coupled effects of atmospheric $\mathrm{N}_{2} \mathrm{O}$ and $\mathrm{O}_{3}$ on the earth's climate. Nature 286: 589-590

World Meteorological Organization (WMO) (1991) Scientific Assessment of Ozone Depletion: 1991. Global Ozone Research and Monitoring Project-Report No. 25 Geneva Switzerland 